\title{
Endoscopic Closure of Transmural Bladder Wall Perforations
}

\author{
Estevao Lima $^{a, b}$, Carla Rolanda ${ }^{a, c}$, Luís Osório $^{b}$, José M. Pêgo $^{a, c}$, David Silva $^{a}$, \\ Tiago Henriques-Coelho ${ }^{f}$, José L. Carvalho ${ }^{f}$, Maria Bergström ${ }^{d}$, Per-Ola Park ${ }^{d}$, \\ Charles A. Mosse ${ }^{e}$, Paul Swain ${ }^{e}$, Jorge Correia-Pinto ${ }^{a, f, *}$ \\ ${ }^{a}$ Life and Health Sciences Research Institute (ICVS), School of Health Sciences, University of Minho, Braga, Portugal \\ ${ }^{\mathrm{b}}$ Department of Urology, Santo António General Hospital, Porto, Portugal \\ ${ }^{\mathrm{c}}$ Departments of Gastroenterology and Anesthesiology, São Marcos Hospital, Braga, Portugal \\ ${ }^{\mathrm{d}}$ Department of Surgery, Sahlgrenska University Hospital/Östra, Gothenburg, Sweden \\ ${ }^{\mathrm{e}}$ Imperial College of Science, Technology and Medicine, London, United Kingdom \\ ${ }^{\mathrm{f}}$ Department of Pediatric Surgery, São Joao Hospital, Porto, Portugal
}

\section{Article info}

\section{Article history:}

Accepted June 3, 2008

Published online ahead of

print on June 13, 2008

\section{Keywords:}

NOTES

Endoscopy

Minimally invasive surgery

Suturing

Bladder

\begin{abstract}
Background: Traditionally, intraperitoneal bladder perforations caused by trauma or iatrogenic interventions have been treated by open or laparoscopic surgery. Additionally, transvesical access to the peritoneal cavity has been reported to be feasible and useful for natural orifice translumenal endoscopic surgery (NOTES) but would be enhanced by a reliable method of closing the vesicotomy.

Objective: To assess the feasibility and safety of an endoscopic closure method for vesical perforations using a flexible, small-diameter endoscopic suturing kit in a survival porcine model. Design, setting, and participants: This pilot study was performed at the University of Minho, Braga, Portugal, using six anesthetized female pigs.

Interventions: Closure of a full-thickness longitudinal incision in the bladder dome (up to $10 \mathrm{~mm}$ in four animals and up to $20 \mathrm{~mm}$ in two animals) with the endoscopic suturing kit using one to three absorbable stitches.

Measurements: The acute quality of sealing was immediately tested by distending the bladder with methylene-blue dye under laparoscopic control (in two animals). Without a bladder catheter, the animals were monitored daily for $2 \mathrm{wk}$, and a necropsy examination was performed to check for the signs of peritonitis, wound dehiscence, and quality of healing.

Results and limitations: Endoscopic closure of bladder perforation was carried out easily and quickly in all animals. The laparoscopic view revealed no acute leak of methylene-blue dye after distension of the bladder. After recovery from anaesthesia, the pigs began to void normally, and no adverse event occurred. Postmortem examination revealed complete healing of vesical incision with no signs of infection or adhesions in the peritoneal cavity. No limitations have yet been studied clinically.

Conclusions: This study demonstrates the feasibility and the safety of endoscopic closure of vesical perforations with an endoscopic suturing kit in a survival porcine model. This study provides support for further studies using endoscopic closure of the bladder which may lead to a new era in management of bladder rupture and adoption of the transvesical port in NOTES procedures.
\end{abstract}

(C) 2008 European Association of Urology. Published by Elsevier B.V. All rights reserved.

\footnotetext{
* Corresponding author. Instituto de Ciências da Vida e Saúde (ICVS), Escola de Ciências da Saúde, Universidade do Minho, Campus de Gualtar, 4709-057 Braga, Portugal. Tel. +351 253604 910; Fax: +351253604831.

E-mail address: jcp@ecsaude.uminho.pt (J. Correia-Pinto).
} 


\section{Introduction}

Intraperitoneal bladder rupture may be caused by trauma (blunt or penetrating) or by iatrogenic interventions, particularly after resection following bladder-tumour removal [1]. Traditionally, these intraperitoneal perforations have been treated by open surgery or recently by laparoscopy, and the results of the prompt repair of the perforation site have been highly desirable, although others have proposed a conservative approach, consisting of either simple bladder drainage or bladder drainage with percutaneous drainage of the peritoneum, which also yields good results [2-4]. But for the majority of authors, urgent surgical closure remains the primary standard treatment for acute intraperitoneal perforation [1,5]. However, surgical closure, whether accomplished by laparotomy or by laparoscopy, is accompanied by risks of slow recovery from surgery because of ileuses and other sequelae, especially in patients who undergo closure of a perforation after laparotomy. Endoscopic closure of iatrogenic or traumatic vesical perforations might have the potential advantage of avoiding major additional trauma associated with laparotomy for surgical closure, with quicker recovery and shorter hospitalization.

Recently, a transvesical approach to the peritoneal cavity has been described by Lima et al [6] as a potential way to perform natural orifice translumenal surgery (NOTES). In experimental studies in pigs it was demonstrated that closure of a 5-mm bladder hole is not absolutely necessary if bladder drainage is assured. However, the development of an effective closure device might enable the widespread adoption of the transvesical port in NOTES.

The aim of this study was to evaluate the feasibility and the safety of endoscopic closure of vesical perforations with an endoscopic suturing kit in a porcine model.

\section{Methods}

The ethical review board of Minho University (Braga, Portugal) approved this study. Survival studies of endoscopic closure of transmural bladderwall perforations using endoscopic suturing kit were performed in six consecutive 20-30-kg female pigs (Sus scrofus domesticus).

\subsection{Pig preparation}

The animals were restrained from food and water for $6 \mathrm{~h}$ before the surgical procedure. As previously described in detail [6], all procedures were performed under general anaesthesia, with 6.0-mm endotracheal intubation (Super Safety clear tracheal tube, Ruschelit, Rüsch, Kernen, Germany) and mechanical ventilation.

\subsection{Endoscopic suturing}

Paul Swain et al $[7,8]$ developed a new device that has proven to be versatile and reliable in a variety of applications. It consists of three components (Fig. 1): (1) 3-0 thread attached to a metal T-tag; (2) a 19gauge needle catheter, a long hollow needle enclosed in a plastic outer sheath, with a stylet to dislodge the T-tag (Cook Endoscopy, WinstonSalem, NC, USA); and (3) a thread lock-and-cut device for the thread (Davol, CR Bard, Murray Hill, NJ, USA).

A metal tag with a 3-0 Monocryl thread (Ethicon, Somerville, NJ) was loaded into the needle, and the needle point was withdrawn into the protective sheath. The needle, loaded with the tag and the thread, was passed through the working channel of a cystoscope, and the needle tip was advanced by a distance that penetrated through the bladder tissue into the peritoneum parietal surface. The depth of suture placement was controlled by extending the needle by a fixed distance in its sheath, which in the current experiments was fixed at $10 \mathrm{~mm}$. The sheath acted as a stop, so that the distance penetrated by the needle could be controlled with some precision. This protective sheath was used to act as a stop to prevent the needle from passing too deeply. Once each pair of stitches had been placed, the threads were locked together. Subsequently, a combination device to lock and cut the thread was used (Fig. 1D-E). First, the threads were passed through holes in a cylinder

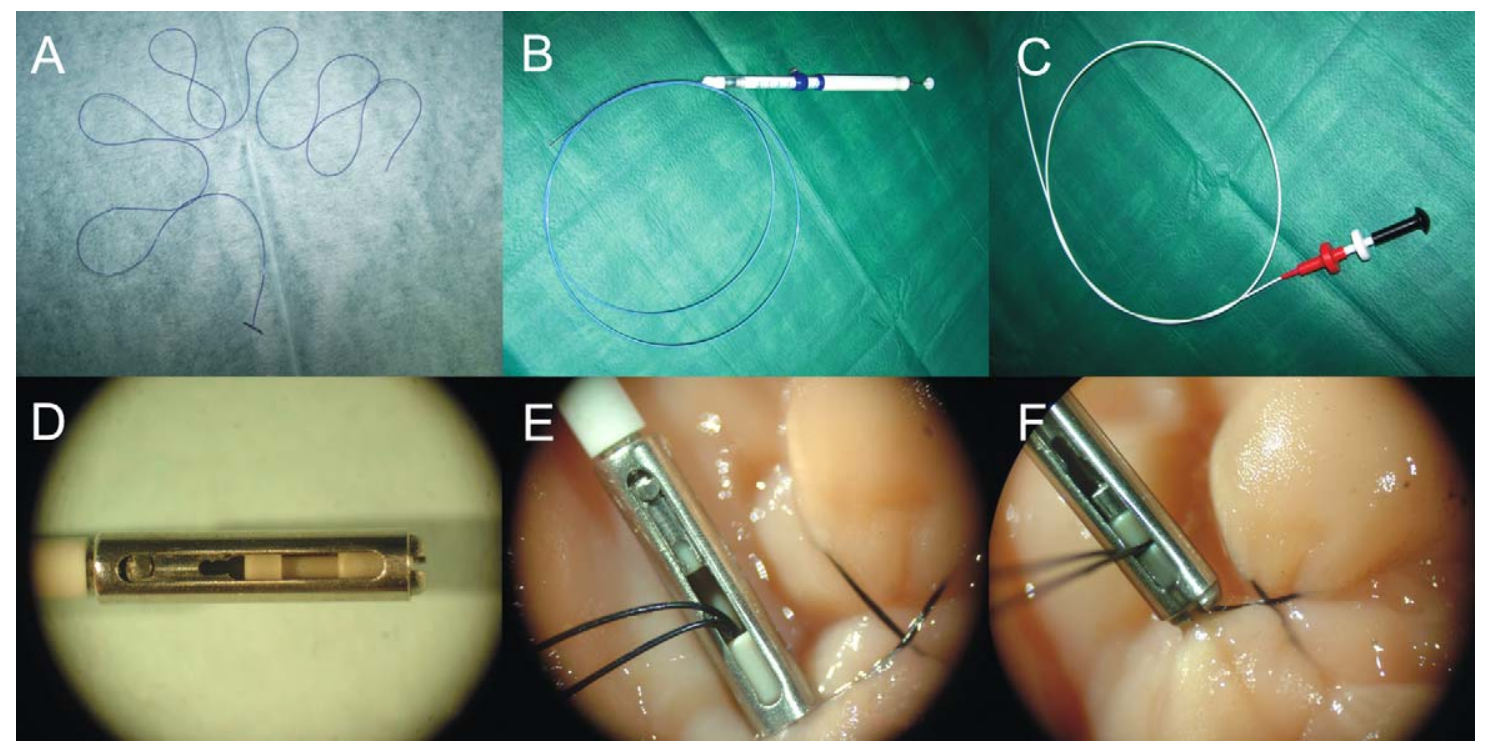

Fig. 1 - Endoscopic suturing kit. (A) The violet Monocryl holding the metallic T-tag; (B) endoscopic needle; (C) locker device; (D-F) sequence of macroimages of the locking mechanism. 


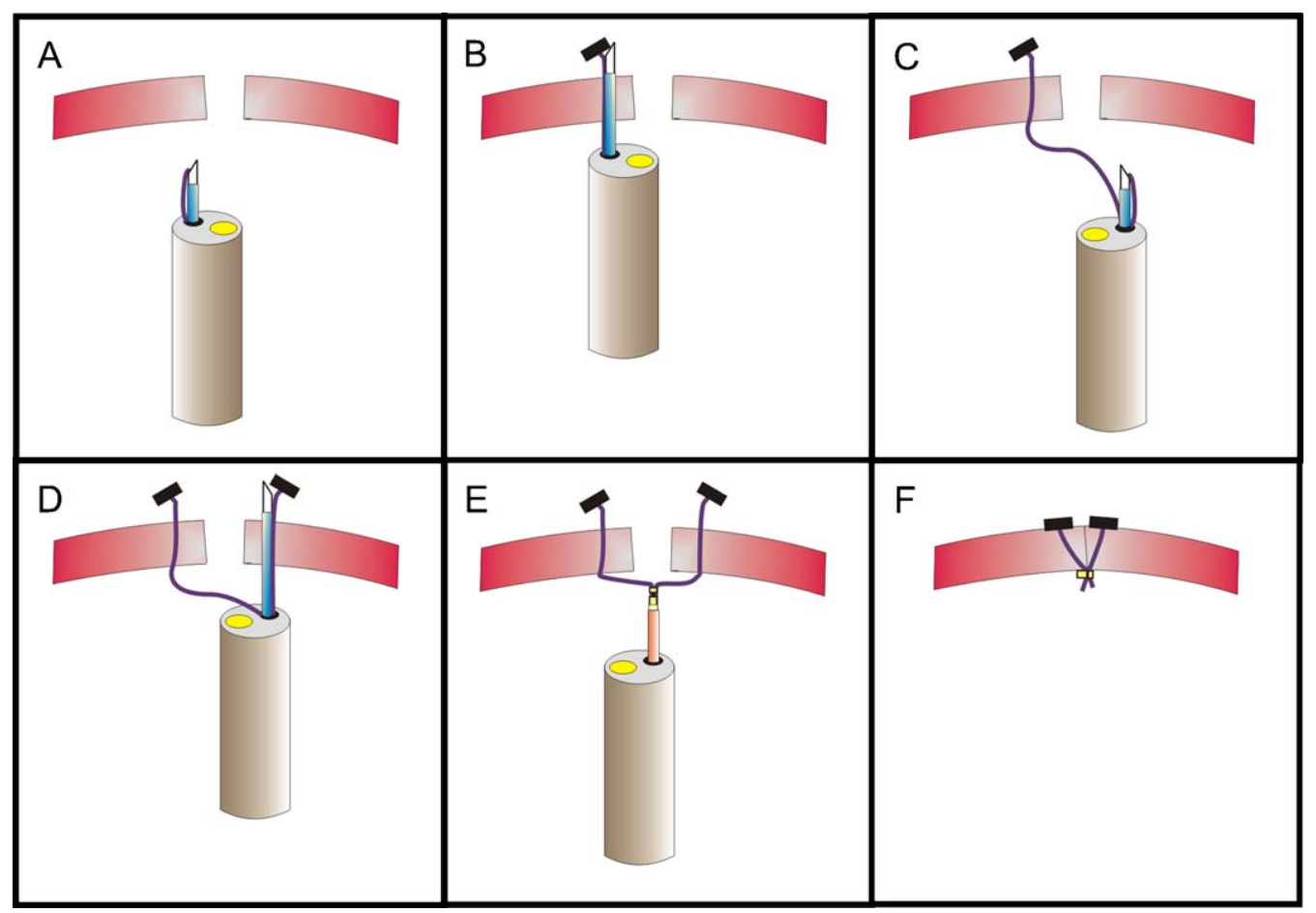

Fig. 2 - Diagram of the T-tags appliance and locking in the bladder wall. (A) Endoscopic needle approaching the edge of vesicotomy; (B) release of the first T-tag; (C) endoscopic needle approaching of the other edge of the vesicotomy; (D) release of the second T-tag; (E) lock advancement; (F) lock release.

with thread locks from a Bard (Davol) EndoCinch kit (Bard Billerica, Boston, MA, USA) and advanced until the lock was positioned snugly against the bladder wall. The lock was closed by pushing a pin into a cylinder compressing the two threads. The lock-and-cut device then forced the threads against an edge, which caused the threads to be cut. The suturing components were small enough to fit through the working channel of a cystoscope.

\subsection{Surgical technique}

A continuous-flow rigid cystoscope (Olympus A20933A with telescope A1931A) was introduced through the urogenital sinus and urethra into the bladder with $\mathrm{CO}_{2}$ distension. Before any further intervention, the bladder was emptied of urine and refilled with $\mathrm{CO}_{2}$. Then a full-thickness longitudinal incision of $10 \mathrm{~mm}$ (four animals) or $20 \mathrm{~mm}$ (two animals) at the bladder dome was created with endoscopic scissors (Karl Storz 26168A) introduced through the working channel of the cystoscope. Subsequently, the cystoscope was introduced into the peritoneal cavity to confirm a full-thickness incision of the bladder wall. All of the animals developed pneumoperitoneum, which was controlled with the insertion of a Veress needle into the peritoneal cavity.

Three steps were involved in the endoscopic closure of the perforation (Fig. 2): (1) With the animals in Trendelenburg position, the needle punctured on the edge of the perforation. (The 19-gauge needle was loaded with the metal T-tag attached to a 3-0 violet Monocryl thread $90 \mathrm{~cm}$ in length was advanced through the working channel of the cystoscope and placed through the full thickness of one the edges of the bladder wall.) By advancing the stylet, the T-tag and thread were released from the needle and left in the exterior part of the bladder. (2) Needle puncture of the opposite edge of the perforation, followed by release of T-tag, was performed in a similar way. (3) Knot tying was then accomplished, followed by suture cutting with a lockand-cut device which was advanced to tie the threads together. The defect was closed by pulling the threads on either side of the incision together until they were snug against the lock and then closing the lock and subsequently cutting the threads with the lock-and-cut device and suture-cutting device. This resulted in a secure closure of the perforation. The adequacy of immediate closure was checked by performing a methylene-blue leak test under laparoscopic control in two animals. In this procedure the bladder was insuflatted with a hydrostatic column of up to $50 \mathrm{~cm} \mathrm{H}_{2} \mathrm{O}$.

All animals were allowed to recover from anaesthesia. No catheter was left in the bladder. Oral feeding was started within $24 \mathrm{~h}$. All animals were evaluated daily for $15 \mathrm{~d}$. Necropsy examination was then performed with particular attention to the site of vesical perforation.

\section{Results}

Six bladder perforations of $10 \mathrm{~mm}$ to $20 \mathrm{~mm}$ in length were performed on six female pigs. All procedures involved in the creation of the vesical hole (cystoscopy, bladder fullthickness incision, and introduction of stitches) were performed without complication. We confirmed the presence of the perforation when we felt the pneumoperitoneum increase and saw the bowel loops inside the peritoneal cavity with the cystoscope.

The needle loaded with the T-tag punctured the bladder wall $5 \mathrm{~mm}$ to $10 \mathrm{~mm}$ from the edge of the perforation, and this component of the procedure was easy, accurate, and reliable. After application of each pair of threads, the margins of the perforations were closed by pulling smoothly on the threads which were positioned outside the cystoscope. Then each pair of threads was held by applying the cylindrical locks (Fig. 3). The application of the lock and its release was easy and quick in all animals. We were able to close all of the perforations effectively using 

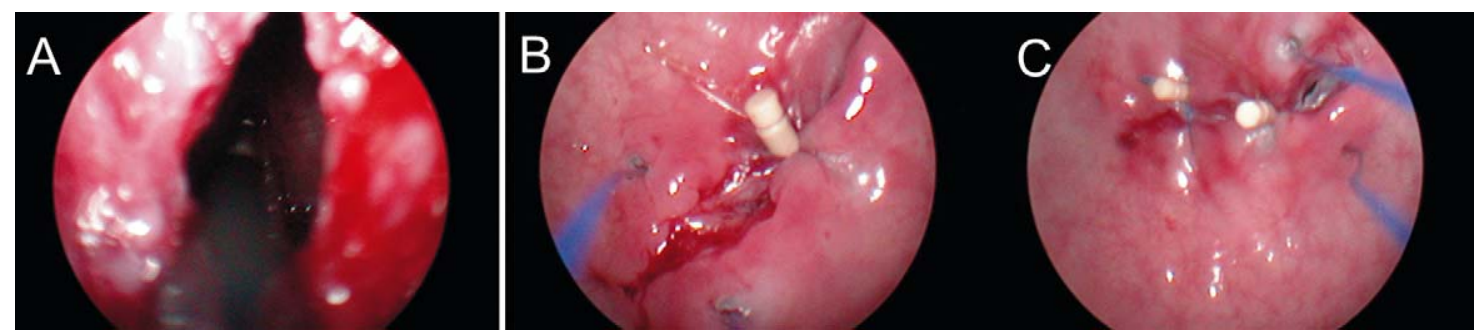

Fig. 3 - Inside bladder view during T-tag application in a 20-mm vesicotomy. (A) Open vesicotomy; (B) One lock applied and the positioning of an additional pair of threads (violet Monocryl) for a subsequent locking; (C) ending endoscopic suture of the 20-mm vesicotomy closure (third pair of T-tags being applied).

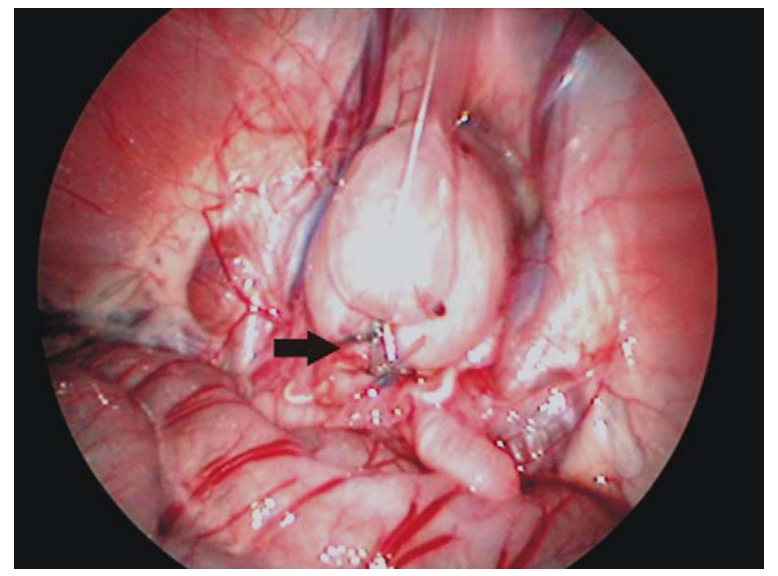

Fig. 4 - Laparoscopic view after T-tag application and distention of the bladder with saline. Twenty-millimeter vesicotomy closure after application of six metallic T-tags (arrow), which allowed three separated stitches.

one pair of threads in perforations $\leq 10 \mathrm{~mm}$ or three pairs in perforations between $10 \mathrm{~mm}$ and $20 \mathrm{~mm}$. From the technical point of view, the endoluminal closure of the bladder perforation using the endoscopic suturing kit was fairly simple and straightforward. By controlling the pneumoperitoneum pressure with intermittent switching, opening or closing the Veress needle introduced into the peritoneal cavity transabdominally, we did not experience any difficulty in maintaining patency of the bladder lumen.
The mean operative time from bladder-wall incision to completion of the closure was around $15 \mathrm{~min}$.

In two animals the entire procedure (perforation, application of T-tags, and locking) was monitored by laparoscopy. In these animals we were able to fill the bladder with saline solution or solution coloured with methylene-blue dye until the bladders were fully distended and no leaks were observed laparoscopically (Fig. 4).

After the surgical procedure no catheters were left in the bladders, and the animals were allowed to recover from anaesthesia. After this, the pigs began to void urine normally and tolerated a regular diet which started the morning after surgery. They ambulated freely, exhibiting normal behaviour for $15 \mathrm{~d}$, and no adverse event occurred during this survival period.

The postmortem examination $15 \mathrm{~d}$ after surgery revealed complete healing of the bladder wall incision. In the locale of vesical perforation, the sutures and locks were detected, and signs of healing without any evidence of transmural dehiscence were observed in all pigs (Fig. 5). There were no signs of infection, visible leakage, or adhesions in the peritoneal cavity of any of the animals.

\section{Discussion}

This study describes a successful method for endoscopic closure of bladder perforations using the endoscopic suturing kit (T-fasteners with a locking cinch). Using a cystoscope with a regular $7 F$ working channel, this method was feasible, easy, and safe in a survival porcine model.

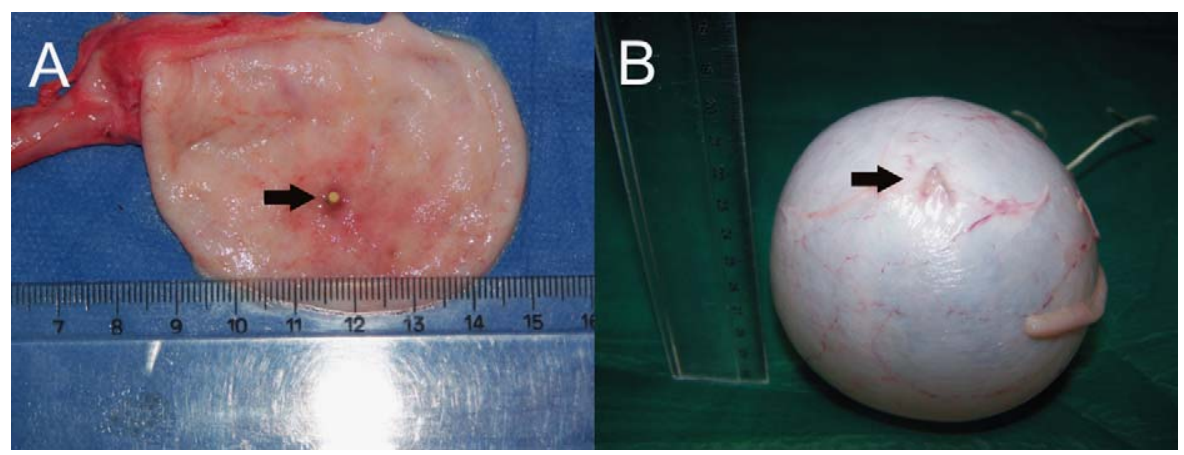

Fig. 5 - Necropsy images from the bladder after closure of a 10-mm vesicotomy. (A) Inside view showing the lock (arrow); (B) outside view showing metallic T-tags (arrow). 
Bladder perforation is a relatively common finding during the clinical activity of a urologist. In fact, several causes can result in bladder perforation, such as iatrogenic damage during transurethral resection of bladder lesions, blunt or penetrating abdomen-pelvic trauma [1,5], and more recently the planned vesicotomy described for NOTES procedures $[6,9,10]$. Until now, if we discovered a bladder rupture after transurethral bladder resection; we needed to convert from endoluminal to laparoscopy or even laparotomy in order to repair the perforation safely [1,5]. For small ruptures, bladder drainage might have been enough, but we commonly needed to leave the bladder catheterized for several days. Also, for small perforations following resections after removal of malignancies, bladder catheterization might have been be contraindicated in order to avoid intraperitoneal spillage of malignant cells. This happened because, until now, urologists have been unable to develop a reliable method for closing these perforations endoluminally.

Interestingly, if we look for endoscopic methods for closing perforations from other viscera like the gastrointestinal tract, it is possible to find many methods and devices designed for this purpose. In fact, one of the most highlighted challenges in surgery and gastroenterology is the endoscopic suturing of intestinal perforations caused by disease, inadvertent injury during endoscopy and endosurgery, and more recently, intentional perforations created for NOTES purposes. Currently, three types of fullthickness suture devices are under evaluation for perforation closure of stomach and colon: two through-theendoscope devices such as the endoscopic suturing kit (Tfasteners with a locking cinch) and the gProx (USGI Medical, San Clemente, CA, USA), and one over-theendoscope device, the Eagle Claw Suturing Device (Apollo Group and the Olympus Medical Systems, Tokyo, Japan) [11]. The endoscopic suturing kit has been used successfully in different gastrointestinal procedures (perforations after endoluminal gastric or colon resections, transgastric gastrojejunostomy and pyloroplasty, closure of perforated gastric ulcer) both in animals and humans [12-17]. Because this method uses equipment that works through a $2.8-\mathrm{mm}$ channel of a standard gastroscope, we hypothesized that we could apply it through a 7F working channel of a cystoscope.

In this study, we applied the endoscopic suturing kit (Tfasteners with a locking cinch) method to closure vesicotomies from $10 \mathrm{~mm}$ to $20 \mathrm{~mm}$ in length, leaving the animals to survive without any bladder catheter. The method to apply the T-tags was easy, quick, and reliable to close a vesicotomy independent of its dimensions. In fact, we could apply one to three T-tags without difficulty in a mean time of $\sim 15 \mathrm{~min}$.

This method uses a full-thickness stitch that was easily applied in the bladder and does not require complex technology. All animals survived without any problems. A potential concern of closure with a full-thickness, needlepuncture technique is the risk of injury to adjacent intraabdominal viscera. To avoid this, the depth of suture placement needs to be controlled precisely by extending the needle by a fixed distance in its sheath according the bladder-wall thickness that, in pigs, we found to be $10 \mathrm{~mm}$. Additionally, such complications can also be avoided using the Trendelenburg position and the pneumoperitoneum that is generated after bladder perforation, since we use $\mathrm{CO}_{2}$ during cystoscopy. These components of the procedure are important in order to avoid inadvertent placement of T-tags into a contiguous bowel loop and may be the reasons that we did not experience any of these complications in the postoperative period.

Regarding the appliance of T-tags in the bladder, it should be emphasized that for us, it seemed even easier than in the stomach or the colon because the instruments involved in the T-tag application and locking worked inside a rigid device (cystoscope) and the puncture was always done perpendicular to the bladder wall. Moreover, we would highlight that this method allowed application of each stitch precisely in place and always under good visual control.

Based on our previous experience with the transvesical approach to the peritoneal cavity using an over-tube (NOTES techniques) that easily allowed control of the pneumoperitoneum, we know that a major concern during the planning of these studies is the potential risk of a massive air leak through the bladder perforation into the peritoneal cavity causing tension on the pneumoperitoneum, resulting in cardiorespiratory compromise. To avoid this, we inserted percutaneously a Veress needle to relieve pneumoperitoneal hypertension. By using this decompression technique immediately after the development of the pneumoperitoneum, none of the animals developed cardiorespiratory compromise, and the visibility of the perforation was maintained for successful closure. Moreover, in practice, we did not observe problems with bladder deflation, even in the pigs that had measured evidence of a pneumoperitoneum before the introduction of Veress needle. Indeed, small, full-thickness, flexible endoscopic incisions in the bladder do not usually collapse the bladder completely. Another advantage of percutaneous insertion of a Veress needle into the peritoneal cavity is the possibility of emptying the peritoneal cavity of $\mathrm{CO}_{2}$ after bladder-wall closure.

A potential limitation of this method that leaves a foreign body in the bladder (the locks) might be the lithogenic stimulus that it can promote. It is well known that any nonabsorbable material left in bladder can start a lithogenic process, and in fact, the locks are made of PEEK (polyetheretherketone), a nonabsorbable material. However, in our experiments we used for the first time T-tags held in place by an absorbable stitch using a 3-0 Monocryl thread. Therefore, we can predict that after 4-6 wk the absorption of the thread releases the lock into the bladder cavity that, because of its small dimensions ( $3 \mathrm{~mm} \times 1.5 \mathrm{~mm}$ ), can be easily voided in the urine through the urethra. The application of T-tags with an absorbable suture is an innovative aspect of this study.

Regarding the potential benefits of this method to close iatrogenic bladder perforations endoscopically, we predict that it may be possible to avoid the additional trauma 
associated with laparotomy for surgical closure, with consequent quicker recovery and shorter hospitalization. The ability to close a perforation immediately within $10 \mathrm{~min}$ to $15 \mathrm{~min}$ after recognition during, for instance, a bladder resection limits the inevitable delay in treatment and may minimize the morbidity associated with surgical trauma. Moreover, it has the additional advantage of avoiding the risk of adhesions that may develop from peritoneal incision and dissection often involved with laparotomy and even laparoscopy. The duration of hospitalization may be shortened by eliminating the risk of ileus that frequently complicates laparotomy. Patients with serious comorbid conditions may not tolerate general anaesthesia and additional trauma associated with the open surgery. This method can be completed with the patient under raquianaesthesia, thereby eliminating the need for general anaesthesia and risks associated with it. Lastly, we predict that the cost-effectiveness of this method when compared with open or laparoscopic methods might constitute an additional advantage for its clinical application. However, although the suturing kit already has FDA (Food and Drug Administration [USA]) and CE (Conformité Européenne [Europe]) approval, it does not yet have a commercially defined price.

Although this study appears to provide enough support for human application, further studies might be useful to define the role of endoluminal closure of bladder perforations in terms of size (small vs large perforations), underlying bladder pathology (normal bladder vs inflamed bladder), and comparison with surgical closure. In contrast to our experiments, where we studied the closure of longitudinal perforations, rupture after trauma or iatrogenic are often not linear but irregular. Although the authors believe that this is not critical or a limitation for the application of T-tags, testing T-tags in these circumstances may be warranted.

Finally, despite the obvious utility of this method in iatrogenic and traumatic bladder rupture, this study strongly encourages application of the transvesical port in the emerging field of NOTES, which in general aims to minimize surgically associated abdominal stress. In fact, after the description of the transvesical port and its usefulness to perform several intra-abdominal procedures (ie, cholecystectomy and nephrectomy), it would be advantageous to develop a reliable closure method for vesicotomy which avoids bladder catheterization $[18,19]$. We believe the observations presented here might be relevant to transluminal procedures performed during NOTES procedures using the transvesical approach.

\section{Conclusions}

This study demonstrates the feasibility and the safety of endoscopic closure of vesical perforations using an endoscopic suturing kit (T-fasteners with a locking cinch) in a survival porcine model. This study provides support for further studies which assess the usefulness of clinical application of T-fasteners in this novel endoscopic method to close perforations in the bladder. This development may open a new era in management bladder rupture and transvesical port in NOTES procedures.

Author contributions: Jorge Correia-Pinto had full access to all the data in the study and takes responsibility for the integrity of the data and the accuracy of the data analysis.

Study concept and design: Lima, Correia-Pinto.

Acquisition of data: Lima, Osório, Pêgo, Silva, Henriques-Coelho, Mosse. Analysis and interpretation of data: Lima, Correia-Pinto.

Drafting of the manuscript: Lima, Correia-Pinto.

Critical revision of the manuscript for important intellectual content: Bergström, Park, Mosse, Swain.

Statistical analysis: None.

Obtaining funding: Lima, Rolanda, Carvalho, Correia-Pinto.

Administrative, technical, or material support: Osório, Silva, HenriquesCoelho.

Supervision: Correia-Pinto.

Other (specify): None.

Financial disclosures: I certify that all conflicts of interest, including specific financial interests and relationships and affiliations relevant to the subject matter or materials discussed in the manuscript (eg, employment/affiliation, grants or funding, consultancies, honoraria, stock ownership or options, expert testimony, royalties, or patents filed, received, or pending), are the following: Paul Swain is a named inventor on patents relating to the devices used in this study. Maria Bergström, Per-Ola Park, Charles A. Mosse, and Paul Swain act as consultants for Ethicon Endosurgery but received no fee for their contribution to this work. The remaining authors have no financial or other interests in manufacture or distribution of any device mentioned in this article. The authors received no consulting fees.

Funding/Support and role of the sponsor: None.

\section{References}

[1] Gomez RG, Ceballos L, Coburn M, et al. Consensus statement on bladder injuries. BJU Int 2004;94:27-32.

[2] Figueiredo AA, Tostes JG, Jacob MV. Laparoscopic treatment of traumatic intraperitoneal bladder rupture. Int Braz J Urol 2007;33: $380-2$.

[3] Yee DS, Kalisvaart JF, Borin JF. Preoperative cystoscopy is beneficial in selection of patients for laparoscopic repair of intraperitoneal bladder rupture. J Endourol 2007;21:1145-8.

[4] Pansadoro A, Franco B, Laurenti C, Pansadoro V. Conservative treatment of intraperitoneal bladder perforation during transurethral resection of bladder tumor. Urology 2002;60:682-4.

[5] Lynch TH, Martínez-Piñeiro L, Plas E, et al. EAU guidelines on urological trauma. Eur Urol 2005;47:1-15.

[6] Lima E, Rolanda C, Pêgo JM, et al. Transvesical endoscopic peritoneoscopy: a novel 5-mm port for intra-abdominal scarless surgery. $\mathrm{J}$ Urol 2006;176:802-5.

[7] Swain P, Park PO. Endoscopic suturing. Best Pract Res Clin Gastroenterol 2004;18:37-47.

[8] Swain P. Endoscopic suturing: now and incoming. Gastrointest Endosc Clin N Am 2007;17:505-20.

[9] Lima E, Rolanda C, Pêgo JM, et al. Transvesical thoracoscopy: a natural orifice translumenal endoscopic approach for thoracic surgery. Surg Endosc 2007;21:854-8.

[10] Gettman MT, Blute ML. Transvesical peritoneoscopy: initial clinical evaluation of the bladder as a portal for natural orifice translumenal endoscopic surgery. Mayo Clin Proc 2007;82:843-5. 
[11] Sclabas GM, Swain P, Swanström LL. Endoluminal methods for gastrotomy closure in natural orifice transenteric surgery (NOTES). Surg Innov 2006;13:23-30.

[12] Ikeda K, Mosse CA, Park PO, et al. Endoscopic full-thickness resection: circumferential cutting method. Gastrointest Endosc 2006; 64:82-9.

[13] Ikeda K, Fritscher-Ravens A, Mosse CA, et al. Endoscopic full-thickness resection with sutured closure in a porcine model. Gastrointest Endosc 2005;62:122-9.

[14] Park PO, Bergström M, Ikeda K, et al. Endoscopic pyloroplasty with full-thickness transgastric and transduodenal myotomy with sutured closure. Gastrointest Endosc 2007;66:116-20.

[15] Bergström M, Ikeda K, Swain P, Park PO. Transgastric anastomosis by using flexible endoscopy in a porcine model (with video). Gastrointest Endosc 2006;63:307-12.
[16] Raju GS, Shibukawa G, Ahmed I, et al. Endoluminal suturing may overcome the limitations of clip closure of a gaping wide colon perforation (with videos). Gastrointest Endosc 2007;65: 906-11.

[17] Pham BV, Raju GS, Ahmed I, et al. Immediate endoscopic closure of colon perforation by using a prototype endoscopic suturing device: feasibility and outcome in a porcine model (with video). Gastrointest Endosc 2006;64:113-9.

[18] Rolanda C, Lima E, Pêgo JM, et al. Third generation cholecystectomy by natural orifices: transgastric and transvesical combined approach. Gastrointest Endosc 2007;65:111-7.

[19] Lima E, Rolanda C, Pêgo JM, et al. Third generation nephrectomy by natural orifices translumenal endoscopic surgery. J Urol 2007;178: 2648-54.

\section{Editorial Comment on: Endoscopic Closure of Transmural Bladder Wall Perforations \\ Richard Naspro \\ Urology Unit, Humanitas Gavazzeni Hospital, \\ Bergamo, Italy \\ nasprorichard@yahoo.com}

Lima and coworkers present a well-conducted safety and feasibility pilot study that was performed using a novel endoscopic kit for the management of bladder wall perforations in a porcine model [1]. The results are of potential relevance as they are presented by authors with robust experience in the transvesical approach to the peritoneal cavity during natural orifice transluminal endoscopic surgery (NOTES). The thrust behind this paper seems to confirm the current trend of the surgical world and, in particular, of urology to find new, minimally invasive, alternative approaches for many surgical procedures [2,3].

As pointed out by the authors [1], the clinical usefulness of this endoscopic kit can be found in the apparently safe achievement of a water-tight bladder closure at the end of surgery; however, striving toward minimally invasive surgery must not distract the clinician from accurate and standard clinical management. In this case, the feeling is that the endoscopic closure of the bladder should be reserved for cases in which a targeted perforation is performed and any other extravesical problem has been previously assessed. When tackling abdominal trauma, for example, a laparoscopic abdominal inspection can be a better investigational tool. Anyhow, this study can indeed lead the way in suggesting an alternative when performing a novel and apparently valid method in a potentially catheter-free setting. As in most cases of uncomplicated bladder perforation, however, simple catheterization can be sufficient for healing. The question of when to use the device remains unanswered. Many points raised by this paper need to be further assessed, such as the cost-benefit ratio, the safety of the procedure, and the rationale and the correct indications for its use [4].

\section{References}

[1] Lima E, Rolanda C, Osório L, et al. Endoscopic closure of transmural bladder wall perforations. Eur Urol 2009;56:151-8.

[2] Branco AW, Branco Filho AJ, Kondo W, et al. Hybrid transvaginal nephrectomy. Eur Urol 2008;53:1290-4.

[3] Box GN, Lee HJ, Santos RJ, et al. Rapid communication: Robot-assisted NOTES nephrectomy: initial report. J Endourol 2008;22:503-6.

[4] Gettman MT, Box G, Averch T, et al. Consensus statement on natural orifice transluminal endoscopic surgery and single-incision laparoscopic surgery: heralding a new era in urology? Eur Urol 2008;53:1117-20.

DOI: 10.1016/j.eururo.2008.06.011

DOI of original article: 10.1016/j.eururo.2008.06.010
Editorial Comment on: Endoscopic Closure of

Transmural Bladder Wall Perforations

Hiten R.H. Patel

Section of Laparoscopic Urology,

Division of Surgery and Interventional Sciences,

University College London Hospital, London,

United Kingdom

hrhpatel@hotmail.com

NOTES (natural orifice transluminal endoscopic surgery) is gaining worldwide attention among the public [1] and interventional doctors [2]. Development of the instruments for this approach are a limiting factor; thus, Lima et al [3] should be congratulated for performing this feasibility study using a novel NOTES endoscopic suturing and cutting instrument (shown in Fig. 1 of their article) following intraperitoneal bladder rupture. This elegant system would appear to have a short learning curve and to be useful in clinical practice; however, as with any novel device, a few caveats need to be addressed.

Assuming a human model is used, there will on occasion be intraperitoneal corruption of varying degrees, including surgery, perforation of the bowel, inflammatory disease, or systemic illness. Although transvesical intra- 
periteonoscopy has been shown to be feasible in humans [4], the above examples would all cause adhesions, making blind entry or manipulation in this area unsafe.

In these situations, when performing laparoscopic surgery, we would advocate a retroperitoneal approach to avoid the corrupted areas [5]. If the surgeon were unfamiliar with this approach, an open technique (Hasson) would be used to safely enter the intraperitoneal space, followed by laparoscopic adhesiolysis.

Another issue would be the blind passing of the needle and thread with clips into the peritoneal cavity, which would increase the risk of bowel injury and potentiate the formation of an enterovesical fistula or bowel leak (see Fig. 1 in MacRae [1]). The clips to hold the suture and the suture itself are foreign bodies, so the risk of stone formation is an issue; however, this future risk is relatively small compared to performing an emergency laparotomy for the perforation.

Finally, if planning on performing this procedure, peritoneal inspection must be performed to reliably inspect the entire length of bowel. A flexible camera could overcome this issue and allow an adequate inspection. Any bowel injury would need suturing. The system described could oversew any areas with diathermy injury or formal enterotomy, but the bowel would need steadying to complete the repair. Currently, the described system cannot perform the latter.

If using this instrument in clinical practice, I would suggest that the patient be placed in steep Trendelenburg, followed by intraperitoneal pneumoinsufflation to further delineate the bladder perforation from any organs, placement of a 5-mm umbilical camera to inspect the bowel in detail, and direct observation of the instrument suturing the perforation. If bowel is affected, two additional 5-mm ports would suffice to safely repair any enterotomies (and if needed, repair the bladder).

I hope to see further development of these instruments as we move forward into the new era of NOTES.

\section{References}

[1] MacRae F. Surgeons remove man's appendix through his mouth in world first operation. Mail Online. April 19, 2008. Available at www.dailymail.co.uk/news/article-560021/Surgeons-removemans-appendix-mouth-world-operation.html.

[2] Gettman MT, Box G, Averch T, et al. Consensus statement on natural orifice transluminal endoscopic surgery and single-incision laparoscopic surgery: heralding a new era in urology? Eur Urol 2008;53:1117-20.

[3] Lima E, Rolanda C, Osório L, et al. Endoscopic closure of transmural bladder wall perforations. Eur Urol 2009;56:151-8.

[4] Gettman MT, Blute ML. Transvesical peritoneoscopy: initial clinical evaluation of the bladder as a portal for natural orifice translumenal endoscopic surgery. Mayo Clin Proc 2007;82: 843-5.

[5] Joseph JV, Patel HR. Extraperitoneal laparoscopy and robotic surgery. New York: Springer. In press.

DOI: 10.1016/j.eururo.2008.06.012

DOI of original article: 10.1016/j.eururo.2008.06.010 